\title{
Endometrial stromal sarcoma presenting as multiple primary tumor
}

\author{
Rajiv Kumar Saxena ${ }^{1}$, Gurpreet Singh Sandhu', Karimbanamalayil Madhavi Babu ${ }^{3}$ \\ ${ }^{1}$ Department of Obstetrics and Gynaecology, Vydehi Institute of Medical Sciences, Bangalore, India \\ ${ }^{2}$ Department of Obstetrics and Gynaecology, Command Hospital Air Force, Bangalore, India \\ ${ }^{3}$ Department of Obstetrics and Gynaecology, 12 Air Force Hospital, Gorakhpur, India \\ Email:drrajivsaxena@yahoo.com
}

Received 27 September 2012; revised 29 October 2012; accepted 7 November 2012

\begin{abstract}
Endometrial Stromal Sarcoma (ESS) is a rare uterine malignancy. The annual incidence of ESS has been reported as 1 - 2 per million women. In the general population approximately 10 percent of cancer patients may develop a second primary cancer. Synchronous multiple primary tumors constitute only $20 \%$ of all multiple primary tumors, and by definition, occur within a period of two month. This case report is presented to document the occurrence of ESS as a "Synchronous Multiple Primary Tumor", which is an extremely rare event. Previously only one case report has documented such an occurrence. The literature is also briefly reviewed. This case underlines the requirement of a thorough initial evaluation for a patient with a gynecological malignancy and a regular follow-up. Follow-up includes patient education regarding symptoms of any recurrence, physical examination repeated at 6 - 12 monthly interval, along with imaging studies when indicated.
\end{abstract}

Keywords: Endometrial Stromal Sarcoma; Synchronous Multiple Primary Tumour; Invasive Ductal Breast Carcinoma

\section{INTRODUCTION}

Endometrial Stromal Sarcoma (ESS) is a rare uterine malignancy. This case report is presented to document the occurrence of ESS as a "Synchronous Multiple Primary Tumor", which is an extremely rare event. Previously only one case report has documented such an occurrence [1].

\section{CASE REPORT}

A 46 yrs. old perimenopausal lady of Indian origin, presented with irregular excessive vaginal bleeding 8 - 10 days every 15 - 25 days since one year. She did not have family history of breast or genital malignancies.
She was under regular treatment for chronic glomerulonephritis and hypertension, since 3 years. Hypertension was well controlled on regular medication with Tab Amlodipine $5 \mathrm{mg}$ HS and Tab Losartan 25 mg BD.

On examination, the patient had BMI of $18.7 \mathrm{Kg} / \mathrm{M}^{2}$. There was no pallor, pedal edema, lymphadenopathy or icterus. Breast examination revealed a small, firm, mobile lump in left breast with dark brown color discharge from nipple. She had a 10-week size uterus, with irregular surface.

Fine Needle Aspiration Cytology (FNAC) from the breast lump revealed ductal carcinoma. She underwent workup for Modified Radical Mastectomy (MRM) by surgical oncologist and she did not return to Gynecology out-patient till one week after MRM surgery.

Her ultrasound evaluation showed a hypo echoic lesion $(53 \times 49 \times 22 \mathrm{~mm})$ arising from posterior wall with intramural extension into the endometrial cavity. The lesion showed increased vascularity on Doppler study, hence a differential diagnosis of fibroid uterus or fibro sarcoma was suggested. Cervical cytology was negative for intraepithelial neoplasia. Diagnostic hysteroscopy had to be abandoned due to poor visualization because of continuous bleeding, however, copious endometrial curetting were obtained on curettage. Histopathology revealed stromal hyperplasia with hyalinization and cellular atypia.

Patient underwent a total abdominal hysterectomy with bilateral salpingo-oophorectomy four weeks after the MRM procedure. Per-operative findings: uterus was 10 weeks size, serosal surface was intact, both ovaries and tubes were normal.

Hysterectomy specimen revealed a polypoidal mass in uterine cavity, measuring $5 \times 3 \times 2 \mathrm{~cm}$ extending into the intramural region. Deapth of invasion was less than 50\% of myometrial thickness. Histology revealed a low-grade endometrial stromal sarcoma, with a Mitotic score of 8 Mitotic Figures (MF) per 10 High Power Fields (HPF) (Figure 1). Lympho-vascular invasion was present. Im- 
mune Histochemistry (IHC) with CD-10 was positive (Figure 2). Other IHC testing like desmin, h-caldesmon, Ki-67, ER/PR was not done. Both ovaries and tubes had normal histology.

Mastectomy specimen (Figure 3) showed a $3.5 \times 2 \times$ $1.5 \mathrm{~cm}$ size invasive ductal carcinoma- $\mathrm{NOS}\left(\mathrm{T}_{2} \mathrm{~N}_{0} \mathrm{M}_{\mathrm{X}}\right)$, ER Status-positive (Allred Score: 3), PR Status-positive (Allred Score: 3), Her2nem-+1 H (negative).

A follow up MRI did not show retroperitoneal lymphadenopathy. Patient is presently asymptomatic and on regular follow-up.

\section{DISCUSSION}

ESS is a rare tumor, which constitutes approximately $10 \%$ of all uterine sarcomas but only around $0.2 \%$ of all

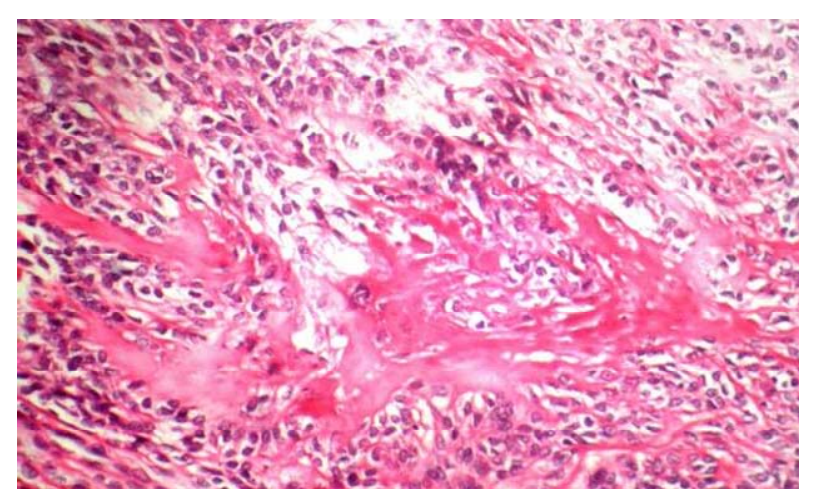

Figure 1. Endometrial Stromal Sarcoma.

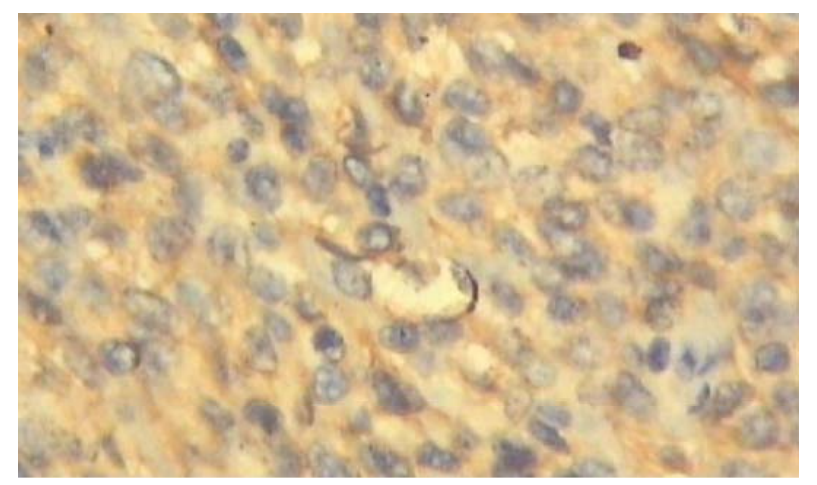

Figure 2. ESS IHC CD-10.

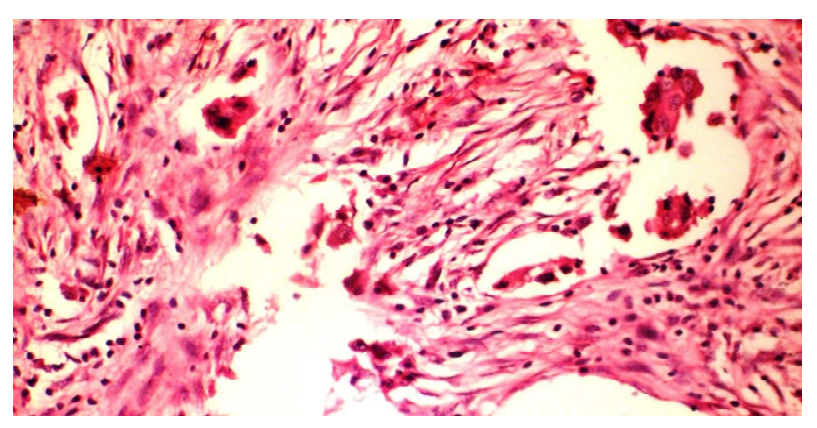

Figure 3. Invasive ductal carcinoma breast NOS. uterine malignancies [2]. This case merits a discussion because of the rarity of its presentation. The annual incidence of ESS has been reported as 1 - 2 per million women [3]. The occurrence of multiple primary cancers is also uncommon. Hence, the occurrence of ESS as a Synchronous Multiple Primary Tumor is an extremely rare event. A web based search retrieved only one reported case of synchronous triple tumors comprising of granular cell tumor of the bladder, endometrial carcinoma and ESS [1].

Uterine sarcomas are rare tumors of mesodermal origin. The three most common histologic variants of uterine sarcoma are ESS, leiomyosarcoma, and malignant mixed müllerian tumor of homologous and heterologous type. Adenosarcoma represents a fourth type of mesenchymal uterine malignancy.

In the general population approximately 10 percent of cancer patients may develop a second primary cancer. A primary cancer is one that originates in a primary site or tissue and is neither an extension, nor a recurrence, nor a metastasis. Multifocal tumours must be excluded. International Association of Cancer Registries (IARC/IACR) rules outline the criteria for diagnosis of multiple primary neoplasm. Multiple primary cancers generally fall into two categories: first being "synchronous", in which the cancers occur at the same time (the Surveillance, Epidemiology, and End Results (SEER) Program definition is within 2 months); and second "metachronous", in which the cancers follow in sequence (more than 2 months apart). When the 2-month rule was applied to the definition of multiple primary cancers, 20 percent of multiple primaries were synchronous and 80 percent were metachronous [4]. Causal mechanisms of multiple primary cancers include genes, the environment, treatment effects, and combinations of these three mechanisms. Family history of cancers, prior treatment with radiotherapy as well as smoking and alcohol history are of significance. The co-morbid condition in our patient was chronic glomerulonephritis and hypertension, which have not been reported to have causal relationship with breast ductal carcinoma or ESS.

Endometrial stromal tumors are composed purely of cells resembling normal endometrial stroma. They are divided into three types on the basis of Mitotic Figures (MF), myometrial and vascular invasion, and observed differences in prognosis: 1) Endometrial Stromal Nodule (ESN); 2) ESS; and 3) High-grade or undifferentiated sarcoma [5]. Distinction between ESN and ESS is based on the findings of irregular tongue like projections of myometrial invasion, greater than $3 \mathrm{~mm}$ and presence of endolymphatic invasion in ESS.

ESS formerly termed low-grade ESS is distinguished from high-grade ESS microscopically by a mitotic rate of less than $10 \mathrm{MF} / 10 \mathrm{HPF}$ as well as clinically by a more 
protracted course. Recurrences typically occur late, and local recurrence is more common than distant metastases. Recurrence occurs in almost one half of cases at an average interval of about 5 years after initial therapy. Treatment includes a total abdominal hysterectomy and bilateral salpingo-oophorectomy. A beneficial effect of radiation therapy has been reported, and pelvic irradiation is recommended only for inadequately excised or locally recurrent pelvic disease. There is also evidence that ESS is hormone dependent or responsive [5].

The National Comprehensive Cancer Network (NCCN) Guidelines for ESS Stage I \& II disease recommends total abdominal hysterectomy and bilateral salpin-gooophorectomy and thereafter only observation and follow-up of the patient. Follow-up includes patient education regarding symptoms of any recurrence, physical examination along with chest, abdomen and pelvic imaging done 3 monthly for 2 yrs., thereafter repeated at 6 - 12 monthly interval [6].

In an epidemiologic study of multiple primary malignnant neoplasms in breast cancer patients from Israel, a significantly higher than expected incidence of second primary cancers occurred at the following five sites: the opposite breast, salivary glands, uterine corpus, ovary, and thyroid. Hence, a patient with a gynecological malignancy requires a thorough initial evaluation and life long follow-up for any other primary malignancy [7].

\section{REFERENCES}

[1] Kiyozuka, Y., Yamamoto, M., Matsuyama, T., Miki, K.,
Tsukamoto, R., Shimano, N. and Tsubura, A. (2006) Synchronous granular cell tumor of the bladder, endometrial carcinoma and endometrial stromal sarcoma. Asia-Pacific Journal of Clinical Oncology, 2, 64-67. doi:10.1111/j.1743-7563.2006.00039.x

[2] Boardman, C.H., Webb, M.J. and Jefferies, J.A. (2000) Low-grade endometrial stromal sarcoma of the ectocervix after therapy for breast cancer. Gynecologic Oncology, 79, 120-123. doi:10.1006/gyno.2000.5893

[3] Pink, D., Lindner, T., Mrozek, A., Kretzschmar, A., Thuss-Patience, P.C., Dörken, B. and Peter, R. (2006) Harm or bene-fit of hormonal treatment in metastatic low-grade endometrial stromal sarcoma: Single center experience with 10 cases and review of the literature. Gynecologic Oncology, 101, 464-469. doi:10.1016/j.ygyno.2005.11.010

[4] Howe, H.L. (2003) A review of the definition for multiple primary cancers in the United States. Workshop Proceedings, Spring-Field (IL): North American Association of Central Cancer Registries, 4-6 December 2002, Princeton.

[5] Harlow, B.L., Weiss, N.S. and Lofton, S. (1986) The epidemiology of sarcomas of the uterus. Journal of the National Cancer Institute, 76, 399-402.

[6] (2012) NCCN clinical practice guidelines in oncology. http://www.nccn.org/professionals/physician gls/pdf/uter ine.pdf

[7] Schenker, J.G., Levinsky, R. and Ohel, G. (1984) Multiple primary malignant neoplasms in breast cancer patients in Israel. Cancer, 54, 145-150. doi:10.1002/1097-0142(19840701)54:1<139::AID-CNC $\underline{\mathrm{R} 2820540129>3.0 . \mathrm{CO} ; 2-\mathrm{U}}$ 